\title{
Original Article Epidemiology of Bovine Mastitis in Selected Major Milk Shade Districts of Arsi Zone, Ethiopia
}

\author{
Behailu Assefa a,* \\ ${ }^{a}$ Department of Veterinary Science, College of Agriculture and Environmental Science, \\ Arsi University, Asella, Ethiopia \\ * Corresponding author. Tel.: +251- 911839999 \\ E-mail address:newbaye@gmail.com, behailuassefaw@gmail.com, \\ behailu@arsiun.edu.et
}

\begin{abstract}
The data for the study on the epidemiology of bovine mastitis were collected from November 2015 to February 2016 to investigate the prevalence of mastitis in administrative towns of three selected districts of Arsi zone, Oromia Regional State, namely Iteya, Sagure, and Asella, Ethiopia; and to analyze the association between the risk factors and mastitis occurrence. Sample cows were selected and examined for clinical mastitis and at the same time, milk samples were evaluated both clinically and using the California Mastitis Test (CMT). Some parameters of the cows which have biological relation with this disease together with other potential risk factors were collected along with primary data collection. The analysis of the primary data showed that overall mastitis prevalence in the locations covered by the study was $28.6 \%$ of which $83.3 \%$ was subclinical, while $16.7 \%$ were clinical mastitis cases. Analysis related to the investigation of the existence of an association between risk factors and mastitis revealed that there was a significant association between the occurrence of mastitis and two of the risk factors evaluated, breed and age of the cow. The other risk factors investigated (i.e. parity, location, and lactation stage had no significant association with mastitis occurrence). Since mastitis is a complex disease that results from the interaction of different factors, it may be difficult to limit it to only some given factors. Environment and management factors and their combination may also have an additional impact on the epidemiology of the disease. A prevalence with the indicated magnitude together with the added effect of a lower level of awareness of the society about milk-associated zoonoses implies that this disease causes significant public health problems. In addition, mastitis may cause silent economic loss through unnoticed production loss along with reduced milk yield from subclinical and clinical (e.g. blind teats) cases. Due to a lack of awareness about mastitis and/or negligence, farmers in all corners of the country are suffering from economic and public health problems imposed by mastitis. This lack of awareness about mastitis-associated problems and/or negligence could be a possible reason for the use of raw milk which is a common scenario particularly in pastoral areas and the selling of adulterated milk which is becoming increasingly practiced in Ethiopia which is also true in these specific localities.
\end{abstract}

Keywords: Bovine; Epidemiology; Mastitis; Prevalence; Risk factors 


\section{INTRODUCTION}

\subsection{Background}

The dairy industry is facing a great set back due to the high prevalence and incidence of mastitis in dairy cows. In addition to causing huge economic losses, this disease also possesses the risk for the transmission of zoonotic diseases, such as tuberculosis, brucellosis, leptospirosis, and streptococcal sore throat. Mastitis is becoming a major health and production constraint in dairy farming. Dairy animals are seen as frequently affected by this disease (Rahmeto et al., 2016).

Up to now, several pieces of research have been conducted but the problem persists (Rahmeto et al., 2016). This may be because of an absence of intensive research that deeply investigates the epidemiology of the disease which can aid in planning to develop tools for effective prevention and/or control measures to be taken and overcome the demand-supply imbalance which becomes a reason for adulteration and deterioration of quality of milk.

\subsection{Problem Statement}

The frequent occurrence of cases of mastitis, reduction in milk yield that does not support the consumer's demand, milk quality problem, and fear of zoonoses (diseases shared by animals and people)

\subsection{Objective}

\section{General Objective}

* To investigate the prevalence of mastitis across the study sites and to identify if there is an association of the disease with risk factors.

\section{Specific Objectives}

* To screen cows with subclinical mastitis and identify cows with clinical mastitis among study animals

* To investigate the prevalence of mastitis across the study sites

* To assess the association of mastitis with some of the host and environmental factors 


\section{METHODOLOGY}

\subsection{Study Area and Study Population}

This observational study on the epidemiology of bovine mastitis was conducted on selected three districts of the Arsi zone which has relatively larger dairy herds, and cows have a higher milk yield compared to other Ethiopian, and that is relatively located close to the school complex, the former School of Agriculture, Adama Science and Technology University. In each of the districts, the study was limited only to their administrative towns which are again potential dairy sites within the districts.

\subsection{Selection and sampling of animals}

The study was designed so that all lactating cows in each of the districts at the time of the survey were included in the sampling process. Besides, dry cows were selected to widen the scope for the investigation of subclinical mastitis. Based on these a total of 126 local and crossbreed cows were sampled. The following parameters were recorded for each animal at the time of sampling; age, breed, parity, stage of lactation, and milk yield.

\subsection{Data collection method}

Data about mastitis among the sampled dairy cows were gathered by collecting a reasonable amount of milk ( $2 \mathrm{ml}$ from each quarter) poured on each of the four plates of the California Mastitis Test (CMT) paddle. The collected milk samples were clinically examined for the presence of abnormalities in milk, such as color change, change in odor, and presence of a clot. The milk was further screened by CMT procedure for subclinical mastitis by adding an equal amount of the CMT reagent, $4 \% \mathrm{NaOH}$ into each of the four shallow cups in the CMT paddle. Clinical mastitis was established by clinical examination of the cow`s udder and teat for gross abnormalities like the asymmetry of an udder and blind teat, before milk sample examination. Examination of milk in case of clinical mastitis can be conducted by visualization of milk for the presence of abnormal flakes. Subclinical mastitis is inapparent during clinical examination of cow and is apparent only during milk analysis. A CMT reagent $(4 \% \mathrm{NaOH}$ in distilled water with an indicator, coloring dye of $1 \%$ bromothymol blue) which is equal in amount to the collected milk sample on each well

of the paddle, was added to the milk. The paddle was tilted and swirled for a moment (15 
seconds) and watched for the formation of a precipitate. Depending on the size of the precipitate, if any or absent, subclinical mastitis is roughly graded as 0 , trace, $+1,+2$, and +3 , where 0 and trace are regarded as negative for subclinical mastitis and the rest are considered as positive for the test, with the level of infection differs in the same order given. Cows having at least one quarter with a CMT score of at least 1+ were defined as positive (NMC, 1999; Quinn et. al., 2004).

The test of subclinical mastitis by the California Mastitis Test (CMT) procedure is an indirect proof test. This test procedure induces the formation of clots in the milk sample after the reagent is added. The clot (precipitate) is formed as a result of the reaction between the added reagent and DNA of somatic cells that may present in milk if there is any inflammation of the mammary gland. An induced clot is formed in milk only when there are somatic cells (white blood cells), even if there are some which may complicate this reaction. These somatic cells are further present in milk only when there is inflammation of the udder which is due to either pathogenic or physical or chemical agents. That is, the presence of a clot in milk implies the presence of somatic cells, and these further imply the presence of an inflammation which in this case is called mastitis. So, based on the presence and level of the clot formed, the test result was interpreted as negative and positive with different levels of positivity which corresponds to the level of somatic cells in milk. $(0=$ 0-200,000 somatic cells per $\mathrm{ml}$ of milk, trace $=150,000-500,000,1=400,000-1,500,000$, $2=800,000-5,000,000,3=>5,000,000$ ) (Miller and Kearns, 1967). The present study was hoping to search for pathogenic agents as the cause of mastitis which are the dominant cause or sometimes maybe the only cause of the disease.

\subsection{Data Analysis}

The data were recorded in the Microsoft Excel spreadsheet and coded before statistical analysis. Descriptive statistics and logistic regression analysis were computed. In the logistic regression analysis, the outcome variable was the presence of mastitis, while the predictors were the different host and environmental risk factors. The association between the dependent variable, cow mastitis status $(0=$ negative and $1=$ positive $)$, and categorical 
independent variables (breed, age, parity, location, and lactation stage) was assessed using logistic regression analyses. All the statistical analyses were performed using R-statistics, declaring significant results at $\mathrm{P}<0.05$. 


\section{RESULTS AND DISCUSSION}

A survey on the epidemiology of bovine mastitis to investigate the magnitude of mastitis among different risk factors and its association with the factors showed the under mentioned results. The study also showed that mastitis was a significant problem for dairy cows in the study area.

\subsection{Prevalence of Mastitis}

Prevalence of mastitis was computed at different levels such as overall prevalence for the total sample from the three study locations. The prevalence for each location, and for the tested risk factors, and also the prevalence of clinical and subclinical mastitis are described in Table 1. The overall prevalence of mastitis was calculated as the proportion of mastitispositive cows (clinical and subclinical) against the total number of cows investigated. A cow was denoted as positive for mastitis if at least a single teat (quarter) with clinical mastitis or CMT positive result was detected.

\section{Table 1: Prevalence of mastitis at a different level of risk factors}

\begin{tabular}{|c|c|c|c|c|c|c|}
\hline $\begin{array}{l}\text { Risk } \\
\text { Factor }\end{array}$ & Category & $\begin{array}{l}\text { No of Cor } \\
\text { Examined }\end{array}$ & $\begin{array}{l}\text { No of Cow } \\
\text { with Mastitis }\end{array}$ & $\begin{array}{l}\text { Prevalence of } \\
\text { mastitis }\end{array}$ & Subclinical & Clinical $\mathrm{P}^{*}$ \\
\hline \multirow[t]{3}{*}{ Location } & Iteya & 18 & 6 & 33.3 & 6 & 0 \\
\hline & Sagure & 21 & 6 & 28.6 & 4 & 2 \\
\hline & Asella & 87 & 24 & 27.6 & 20 & 4 \\
\hline \multirow[t]{2}{*}{ Breed } & Local & 22 & 2 & 9.1 & 2 & 0 \\
\hline & Cross & 104 & 34 & 32.7 & 28 & 6 \\
\hline Lactation & $<3 \mathrm{mth}$ & 21 & 12 & 57.1 & 6 & 6 \\
\hline \multirow[t]{4}{*}{ Stage } & {$[3,6) \mathrm{mth}$} & 9 & 0 & 0 & 0 & 0 \\
\hline & {$[6,9) \mathrm{mth}$} & 33 & 6 & 18.2 & 6 & 0 \\
\hline & {$[9,12) \mathrm{mth}$} & 18 & 9 & 50.0 & 9 & 0 \\
\hline & $>=12 \mathrm{mth}$ & 45 & 9 & 20.0 & 9 & 0 \\
\hline Parity & 1 & 36 & 9 & 25.0 & 7 & 2 \\
\hline \multirow[t]{3}{*}{ Number } & 2 & 32 & 8 & 25.0 & 8 & 0 \\
\hline & 3 & 33 & 11 & 33.3 & 9 & 2 \\
\hline & $>=4$ & 25 & 8 & 32.0 & 6 & 2 \\
\hline \multirow[t]{3}{*}{ Age } & {$[3,6)$ year } & 33 & 6 & 18.2 & 6 & 0 \\
\hline & {$[6,9)$ year } & 72 & 21 & 29.2 & 21 & 0 \\
\hline & $>=9$ year & 21 & 9 & 42.9 & 3 & 6 \\
\hline \multicolumn{5}{|c|}{ Prevalence $(\%)$} & 23.8 & 28.6 \\
\hline
\end{tabular}




\subsection{Association of Mastitis with Risk Factors}

In the logistic regression analyses conducted, statistical significance was set at $\mathrm{P}<0.05$. Several cow-level and environmental factors like the breed of the cow, age of the cow, parity number of the cow, location (study site), and lactation stage of the cow were considered in the logistic regression analysis for the presence of their association with mastitis. Among those factors, the breed was found to be significantly $(P<0.05)$ associated with the presence of mastitis while age had a significant statistical association with mastitis just at 0.05 indicating a slight association. On the other hand, parity, location, and lactation stage did not have a significant effect $(p>0.05)$ on the occurrence of mastitis.

Excluding the non-significant factors and rerunning the logistic regression only for the significant risk factors, breed and age, yield the following out put (Table 2).

Table 2: Multivariable Regression Analysis for the Potential Risk Factors of Mastitis

\begin{tabular}{|c|c|c|c|c|}
\hline & Estimate & std. Error $\mathrm{z}$ value & $\operatorname{Pr}(>|z|)$ & \\
\hline (Intercept) & -5.1638 & $1.6602-3.110$ & 0.00187 & $* *$ \\
\hline Age & 0.6250 & 0.3196 & 0.05050 & . \\
\hline Breed & 1.6117 & 0.7761 & 0.03785 & $*$ \\
\hline
\end{tabular}

Therefore, the regression equestion, that is the final regression model in this case become:

$\operatorname{Ln}($ odd of mastitis $)=-5.1638+1.6117 *$ Breed $+0.6250 *$ Age

But the Logistic regression analysis of the effect of breed alone on the occurrence of mast itis showed that mastitis occurrence was significantly influenced $(p<0.05)$ by the breed $o$ $f$ the cow. The chance of occurrence of mastitis is significantly higher in crossbred cows $t$ han in local breed cows. Mastitis in crossbreed cows increases 4 times than in local breed $\mathrm{s}$. The odds ratio for the breed is 4.857143, which is $\exp (1.5805)=4.857384$ (Appendices) $\operatorname{Ln}($ odd of mastitis $)=-3.8830+1.5805 *$ Breed 
There is also a significant effect of the age of cows alone on the incidence of mastitis. Because the p-value for age is 0.05365 which is approximately equal to 0.05 level of significance.

\section{$\operatorname{Ln}($ odd of mastitis $)=-2.1069+0.6081 *$ Age}

For every one-year increase in age of the cow, the odds of incidence of mastitis increases by 83.7 units. Because $\exp (0.6081)=1.836938$ (Appendices). This implies that the age of the cow has a significant effect on the incidence of mastitis, which means that when the age 0 $f$ the cow increases by one year, the odds of incidence of mastitis increases by 84 units. $T$ hat means there is a positive relationship between the age of cow and odds of incidence of mastitis (also seen from the logistic regression model, positive coefficient of age). The od ds of getting mastitis increase by $84 \%$ as the age of cow increase by one unit (year).

There were different researches conducted so far in Ethiopia on mastitis. The research output of some of these researches was consistent with the present study while others were not. Beyene et al., 2017 have shown that at the analysis of chi-square and univariable logistic regression, four factors namely breed, lactation status, age and number of parity were statistically significant while at multivariable logistic regression breed, age and lactation status showed to be the main risk factors that contribute for occurrence of mastitis.

Rahmeto et.al., 2016 have noted that in the multivariable logistic regression model, breed, parity, and stage of lactation have a significant effect on mastitis occurrence. The occurrence of bovine mastitis and lactation stage was significantly $(\mathrm{p}<0.05)$ associated. That is, higher infection in cows in early lactation stage followed by late and medium lactation stages. Mastitis prevalence was found to be higher in early lactation and lower in mid-lactation stages. The early lactation stage infection might be due to the carryover of infection from the dry period. Rahmeto et al., 2016 also showed that animals over 8 years old were more frequently affected with mastitis and those younger than 5 years were rarely affected. Mastitis is more common and has a serious impact on older lactating animals than in first lactation heifers Also the highest prevalence of mastitis was observed in animals with parity of more than 6 , followed by 3-6 and 1-2 parity. 
Mulugeta and Wassie, 2013 also found that all the potential risk factors considered for a univariate logistic regression, i.e. age, parity, breed, and lactation stage were found significant $(\mathrm{P}<0.05)$. However, age became insignificant when tested with multivariate logistic regression. The prevalence of mastitis varied significantly $(\mathrm{p}<0.05)$ among breeds. It was also appreciated that the lactation stage and parity significantly $(\mathrm{p}<0.05)$ influenced the occurrence of mastitis. 


\section{CONCLUSIONS AND RECOMMENDATIONS}

The frequent occurrence of cases of mastitis, reduction in milk yield that does not support the consumer's demand, milk quality problem, and fear of zoonoses are some of the problems that call attention for the research. The source of mastitis infection may be regarded as contagious or environmental. Contagious pathogens spread during milking. Environmental agents are opportunistic invaders from the cow's environment.

Prevalence and distribution of mastitis might be due to differences in the breeds of cattle reared, age of the animal, parity, the geographical locations, and lactation stage. These variables were subjected to logistic regression analysis and accordingly, the logistic regression model revealed that the breed of the cow among the variables entered remained significant predictors of mastitis in the cows $(p<0.05)$. Age of the cow is slightly significant $(p=0.05)$. The remaining factors analyzed didn't show statistical significance at the 0.05 significance level.

Mastitis causes huge economic losses and also possesses the risk for the transmission of zoonotic agents. It is one of the diseases that cause silent public health and economic loss. Unless preventive and other control measures were in place it may continue to negatively influence human and other animals' health. The big problem in Ethiopia is the absence of clear and comprehensive and frequently updated epidemiological data on animal and human diseases that aid in designing an effective control measure. This is true for mastitis as well.

There should be a comprehensive and frequently updated epidemiological data on mastitis in the study area for that matter in Ethiopia as a whole

* The epidemiological data obtained regularly should be properly analyzed and lead for action

* Evaluation and monitoring measures should always be in place for every control measure chosen and effected in every situation 
* A method in which information-based amendments for the already effected control measure and initiation of new control measure based on need should be in place to control mastitis

Awareness creation and frequent communication of information on mastitis among stakeholders should be implemented

\section{Conflict of Interests}

The author declares that there is no conflict of interest regarding the publication of this paper.

\section{Acknowledgments}

I would like to thank all those individuals and an organization who have got significant contributions to this particular work either directly or indirectly. Particularly, my gratitude goes to the former Adama Science and Technology University School of Agriculture for the financial support for the project. 


\section{REFERENCES}

1. Beyene and Tadele Tolosa (2017): Epidemiology and Financial Impact of Bovine Mastitis in an Animal Production and Research Center and Small Holder Dairy Farms in HoroGuduru Wollega Zone, Western Ethiopia. Journal of Dairy, Veterinary \& Animal Research. Volume 5 Issue 4 - 2017.

2. FAO. (2014). Impact of mastitis in small scale dairy production systems. Animal Production and Health Working Paper. No. 13. Rome.

3. Mulugeta Y. and Wassie M. (2013). Prevalence, risk factors and major bacterial causes of bovine mastitis in and around Wolaita Sodo, Southern Ethiopia. Academic Journals. Vol. 7(48), pp. 5400-5405, 4. DOI: 10.5897/AJMR2013.6261. ISSN 1996-0808

4. NMC, National Mastitis Council (1999). Laboratory Handbook on Bovine Mastitis. revised ed. Madison, Wisconsin: Natl Mastitis Counc, Inc.,:1-30.

5. Quinn J., Carter E., Markey B., Carter R. (2004). Clinical Veterinary Microbiology. London Wild life Publisher. pp. 95-101.

6. Rahmeto A., Hagere H., Mesele A., Bekele M. and Kassahun A. (2016): Bovine mastitis: prevalence, risk factors and isolation of Staphylococcus aureus in dairy herds at Hawassa milk shed, South Ethiopia. BMC Veterinary Research, BMC series.

7. Miller D. D. and Kearns J. V. (1967). Effectiveness of the California Mastitis Test as a Measurement of the Leucocyte Content of Quarter Samples of Milk. Dairy Department, New Mexico State University, Las Cruces 


\section{APPENDICES}

\section{Logistic regression analysis for the association between mastitis and breed of the cows and Odds ratio for breed}

$>$ fit<-glm(Infection Breed,family=binomial(link="logit"),data=gesho)

$>$ summary(fit)

Call:

glm $($ formula $=$ Infection $\sim$ Breed, family $=$ binomial $($ link $=$ "logit" $)$, data $=$ gesho)

Deviance Residuals:

Min 1Q Median 3Q Max

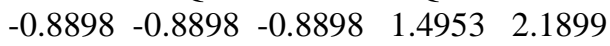

Coefficients:

Estimate Std. Error $\mathrm{z}$ value $\operatorname{Pr}(>|\mathrm{z}|)$

(Intercept) $-3.8830 \quad 1.4976 \quad-2.593 \quad 0.00952 * *$

$\begin{array}{lllll}\text { Breed } & 1.5805 & 0.7704 & 2.052 & 0.04021\end{array}$ *

$---$

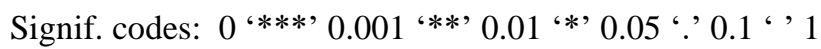

(Dispersion parameter for binomial family taken to be 1)

Null deviance: 150.76 on 125 degrees of freedom

Residual deviance: 144.86 on 124 degrees of freedom

AIC: 148.86

Number of Fisher Scoring iterations: 4

$>\exp (1.5805)$

[1] 4.857384

\section{Logistic regression analysis for the association between mastitis and age of the cows} and Odds ratio for age

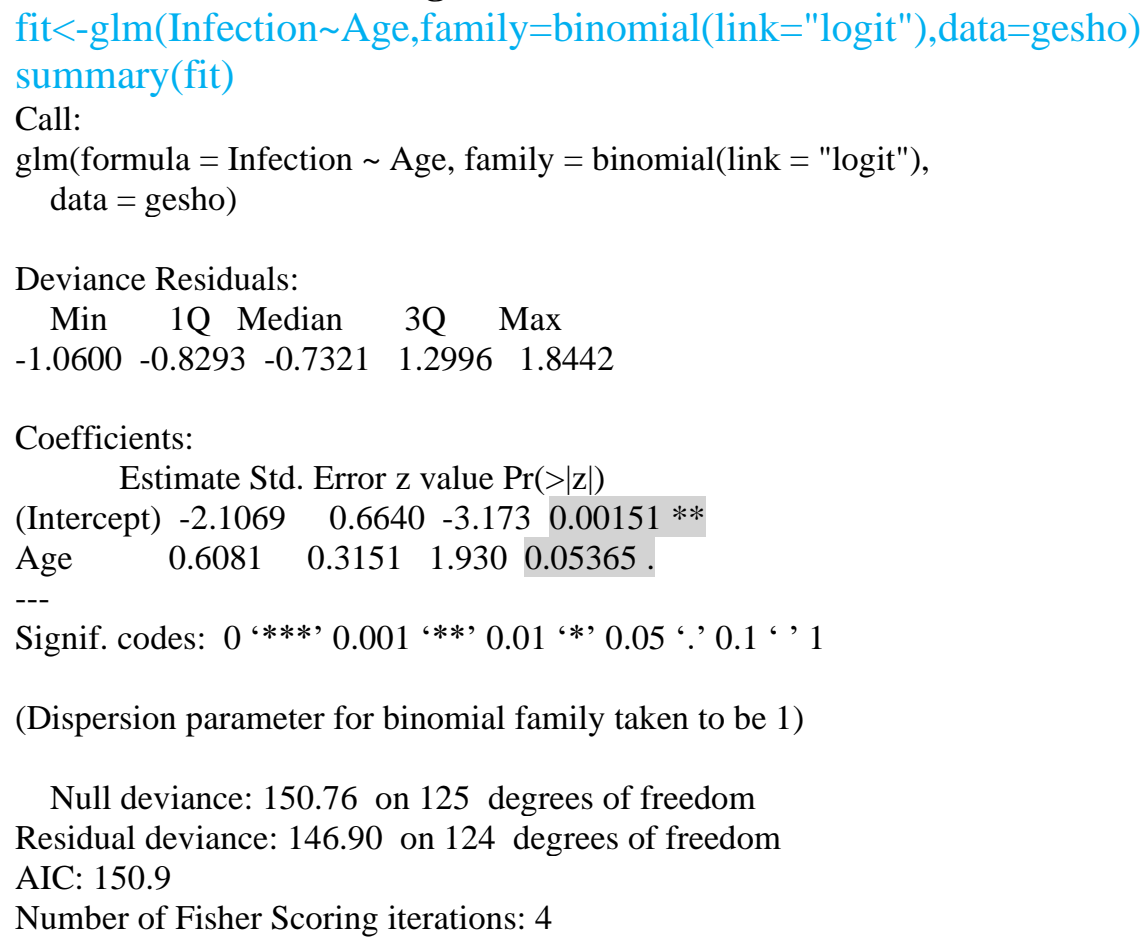


$>\exp (0.6081)$

[1] 1.836938

3. Logistic regression analysis for the association between mastitis and all the risk factors

$>$ fit<-glm(Infection $\sim$ Age+Breed+Lactation+Parity+Location,family=binomial(link="logit"), data=gesho)

$>$ summary(fit)

Call:

glm $($ formula $=$ Infection $\sim$ Age + Breed + Lactation + Parity +

Location, family = binomial $(\operatorname{link}=$ "logit"), data = gesho $)$

Deviance Residuals:

Min 1Q Median 3Q Max

$\begin{array}{lllll}-1.3801 & -0.8841 & -0.6564 & 1.1605 & 2.1921\end{array}$

Coefficients:

Estimate Std. Error z value $\operatorname{Pr}(>|z|)$

(Intercept) $-4.73753 \quad 1.88514 \quad-2.513 \quad 0.0120 *$

$\begin{array}{lllll}\text { Age } & 0.62735 & 0.32359 & 1.939 & 0.0525\end{array}$.

$\begin{array}{llllll}\text { Breed } & 1.58463 & 0.78831 & 2.010 & 0.0444\end{array}$

$\begin{array}{llllll}\text { Lactation } & -0.05195 & 0.14166 & -0.367 & 0.7138\end{array}$

$\begin{array}{lllll}\text { Parity } & 0.09269 & 0.18808 & 0.493 & 0.6221\end{array}$

Location $\quad-0.16765 \quad 0.28832 \quad-0.581 \quad 0.5609$

---

Signif. codes: 0 ‘***’ 0.001 '**’ 0.01 '*’ 0.05 ‘' 0.1 ‘ ' 1

(Dispersion parameter for binomial family taken to be 1)

Null deviance: 150.76 on 125 degrees of freedom Residual deviance: 140.08 on 120 degrees of freedom AIC: 152.08

Number of Fisher Scoring iterations: 4

\section{Odds ratio for the risk factors for mastitis (STATA output)-for comparison with R}

logistic infection age breed lactation location parity

Logistic regression $\quad$ Number of obs $=126$

LR $\operatorname{chi} 2(5)=10.68$

Log likelihood $=-70.042061 \quad$ Pseudo R2 $\quad 0.0708$

\begin{tabular}{|c|c|c|c|c|c|c|}
\hline infection & Odds Ratio & Std. Err. & $\mathrm{Z}$ & $\mathrm{P}>|\mathrm{z}|$ & [95\% Conf. & Interval] \\
\hline age & 1.872646 & 6059864 & 1.94 & 0.053 & 9931339 & 3.531048 \\
\hline breed & 4.877469 & 3.846169 & 2.01 & 0.044 & 1.039854 & 22.87793 \\
\hline lactation & .9493721 & .1344852 & -0.37 & $\begin{array}{ll}7 & 0.714\end{array}$ & .7192135 & 1.253185 \\
\hline location & .8456531 & .2438246 & -0.58 & 0.561 & .4805824 & 1.488047 \\
\hline parity | & 1.097124 & .2063574 & 0.49 & 0.622 & .7588462 & 1.586199 \\
\hline _cons & .0087602 & .0165182 & -2.51 & 0.012 & .0002175 & .3528054 \\
\hline
\end{tabular}

